\title{
Vibration-Based Loosening Detection of a Multi-Bolt Structure Using Machine Learning Algorithms
}

\author{
Oybek Eraliev ${ }^{1}\left(0\right.$, Kwang-Hee Lee ${ }^{2}$ and Chul-Hee Lee ${ }^{2, *}$ \\ 1 Future Vehicle Engineering Department, Inha University, 100 Inharo, Mitchuholgu, Incheon 22212, Korea; \\ oybekeraliev7@gmail.com \\ 2 Mechanical Engineering Department, Inha University, 100 Inharo, Mitchuholgu, Incheon 22212, Korea; \\ gwanghee.yee@gmail.com \\ * Correspondence: chulhee@inha.ac.kr
}

check for updates

Citation: Eraliev, O.; Lee, K.-H.; Lee, C.-H. Vibration-Based Loosening Detection of a Multi-Bolt Structure Using Machine Learning Algorithms. Sensors 2022, 22, 1210. https:// doi.org/10.3390/s22031210

Academic Editor: Mohammad Noori

Received: 7 January 2022

Accepted: 2 February 2022

Published: 5 February 2022

Publisher's Note: MDPI stays neutral with regard to jurisdictional claims in published maps and institutional affiliations.

Copyright: (C) 2022 by the authors. Licensee MDPI, Basel, Switzerland. This article is an open access article distributed under the terms and conditions of the Creative Commons Attribution (CC BY) license (https:// creativecommons.org/licenses/by/ $4.0 /)$.

\begin{abstract}
Since artificial intelligence (AI) was introduced into engineering fields, it has made many breakthroughs. Machine learning (ML) algorithms have been very commonly used in structural health monitoring (SHM) systems in the last decade. In this study, a vibration-based early stage of bolt loosening detection and identification technique is proposed using ML algorithms, for a motor fastened with four bolts $(\mathrm{M} 8 \times 1.5)$ to a stationary support. First, several cases with fastened and loosened bolts were established, and the motor was operated in three different types of working condition (800 rpm, $1000 \mathrm{rpm}$, and $1200 \mathrm{rpm}$ ), in order to obtain enough vibration data. Second, for feature extraction of the dataset, the short-time Fourier transform (STFT) method was performed. Third, different types of classifier of ML were trained, and a new test dataset was applied to evaluate the performance of the classifiers. Finally, the classifier with the greatest accuracy was identified. The test results showed that the capability of the classifier was satisfactory for detecting bolt loosening and identifying which bolt or bolts started to lose their preload in each working condition. The identified classifier will be implemented for online monitoring of the early stage of bolt loosening of a multi-bolt structure in future works.
\end{abstract}

Keywords: bolt loosening; loosening detection; machine learning; bolt-loosening identification; vibration; signal processing

\section{Introduction}

A bolt joint is one of the most important methods for connecting structural components in engineering fields and can be assembled and reused. However, one of the main drawbacks of threaded fasteners is the loosening that occurs under shock or vibration conditions [1]. The loosening can cause a structure to become damaged seriously, so boltloosening detection and identification before failure of the structure are some of the most important issues in engineering. Detection and identification of bolt loosening can, not only keep a structure from experiencing accidents or failure, but also reduce maintenance costs. Therefore, the detection of bolt loosening in many fields of mechanical, aerospace, and civil engineering has been an important topic among many researchers in the last decade.

Generally, the detection techniques for bolt loosening can be divided into three groups. The first is the group of in situ inspection techniques, which include visual inspection by an experienced inspector or use of mechanical devices such as a torque wrench and hammer [2]. Despite the advantages of visual inspection by an expert, which is the simplest and lowest-cost method, it is complicated to detect the early-stages of bolt loosening. In other words, the visual inspection method is better as a way to detect bolts which are totally loosened. However, the hammer impact method is more effective to detect early-stage of bolt loosening than visual inspection, and bolt loosening can be detected easily via the sound that is made when hitting something with a hammer by an experienced inspector 
or using ML algorithms [3]. Although the group of in situ inspection techniques are quite simple and low-cost monitoring techniques, they still have challenges [4]. For instance, one of the main drawbacks of the hummer impact technique is that environmental noise can reduce the accuracy, and the technique is time consuming in some applications that include many bolts, such as bridges [2].

The second group includes computer vision-based techniques that detect bolt-loosening by use of cameras or digital images [5-8]. This group of techniques is unique, with advantages that can overcome the drawbacks of the in situ inspection techniques such as environmental noise and being time consuming. Many researchers have contributed to the development of computer vision-based techniques to detect bolt loosening. For example, Zang et al. [9] carried out a study on bolt-loosening detection using deep learning for a multi-bolt connection. Their model solves two problems: (1) a bolt detection with a convolutional neural network (CNN), and (2) a regression problem to predict the amount loosening of the bolt with a faster region-based convolutional neural network (Faster R-CNN).

Bolt loosening was detected by measuring the rotation angle of a bolt or nut via deep learning in a study by Zhao et al. [10]. They used a set of images taken with a smartphone as a dataset for detection of a bolt head and a number that was written on top of the bolt. The coordinates of the detection boxes of the bolt head and number were used to find the center points of the boxes, and the rotation angle of the bolt was computed using the center points. Valuable investigations have been performed by researchers with this technique. However, there are still some challenging issues. For example, detecting the early stages of the loosening of a bolted joint is very difficult, and in some real conditions, such as those in vehicle engines and turbines, fixing a camera to an appropriate place to detect loosening is complicated. A CNN is also often used as a tool in this technique and has a high computational cost.

The third group includes sensor-based techniques, which include the vibration-based method [11,12], acoustoelastic effect-based method [13,14], piezoelectric sensor-based methods [15,16], and impedance-based method [17,18]. Sensor-based techniques have some unique advantages that can overcome the drawbacks of in situ inspection and computer vision-based techniques such as environmental noise impact, being time consuming, as well as the detection of early-stage bolt loosening. However, sensor-based techniques require fixed sensors and a high-cost system, while the accuracy of the techniques is significantly greater than that of the other techniques that have been described above, and they are reliable [2]. Computer vision-based techniques for bolt loosening detection of multibolt connections have been proposed by several researchers, while a few studies based on vibration-based methods, with use of ML algorithms, for early stage bolt loosening detection and identification have been carried out [19].

Therefore, one of the most popular sensor-based techniques, a vibration-based method, was chosen in this study. This paper proposes the classification and identification of early stages of bolt loosening in a multi-bolt structure using ML classifiers. The application is an AC Motor. For this, 16 cases (healthy and unhealthy conditions) were established, and vibration data were gathered in three operating conditions of the motor $(800 \mathrm{rpm}$, $1000 \mathrm{rpm}$, and $1200 \mathrm{rpm}$ ). A short-time Fourier transformation was conducted to obtain features of the row vibration data. Finally, ML classifiers were evaluated to determine the best classifier, and the classifier that had the greatest accuracy was identified.

The results prove that the determined classifier can detect, not only the early-stages of loosening, but can also identify which bolt or bolts have started to loosen. The theoretical background of signal processing, feature extraction of vibration data for ML classifiers, and ML algorithms are described in Section 2. Section 3 explains the experimental setup in detail. The results and contributions of the study are discussed in Section 4. The conclusions are presented in Section 5. 


\section{Theoretical Background and Signal Processing}

A flowchart of the method proposed in this study is shown in Figure 1. Initially, data acquisition is performed on a motor that has four M8 bolts. Then, short-time Fourier transform (STFT) is applied to extract features of the acquired vibration data. Following this, the extracted dataset is divided into a training dataset for training a model of classifiers and a test dataset for evaluating the accuracy of the models. Finally, ML classifiers are evaluated to determine the best classifier, and the classifier that has the best performance is identified.

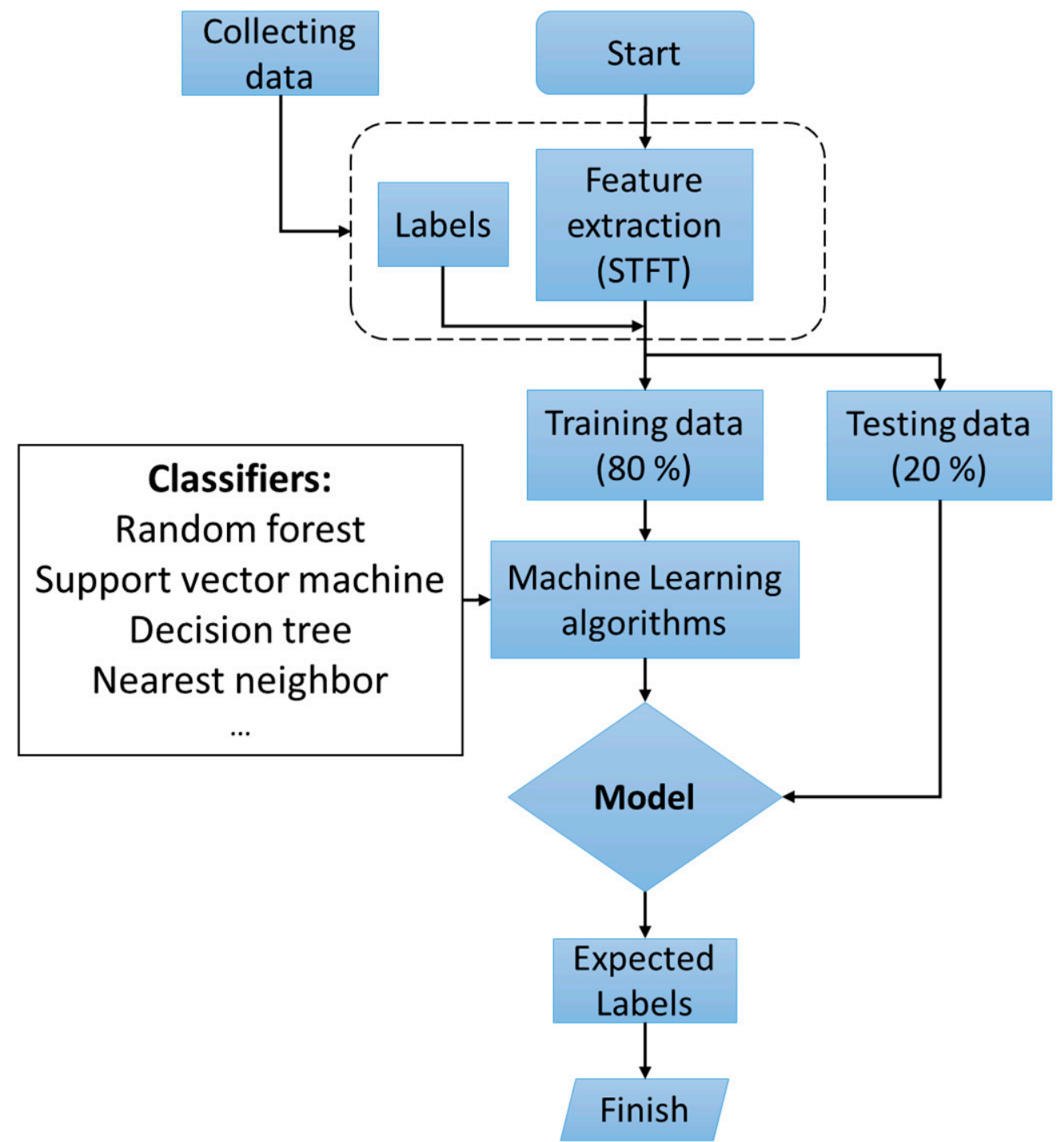

Figure 1. The flowchart of the proposed method for bolt-loosening detection.

\subsection{Feature Extraction (STFT)}

Feature extraction can play a key role in obtaining a reliable result in the ML field. Feature extraction of a row vibration signal can be done in several ways. For example, the fast Fourier transform (FFT) method computes the discrete Fourier transform (DFT) of a data sequence. Fourier analysis transforms a signal from its original domain (typically time or space) to a frequency domain representation, or conversely [20]. Chen et al. [21] used a FFT method in their study for identification and classification of a gearbox fault. S. Ma et al. [22] also used FFT in their study for fault diagnosis of a rotor and bearing. However, performing the FFT on a time-domain signal can give the overall frequency components for the entire time-domain signal. Therefore, it cannot provide information 
about how the frequency is changing over time. To understand, for example, where the high and low pitches are in a vibration signal, the STFT can be applied. It can give a FFT that changes with time. This is not readily apparent from only applying the FFT to the entire time-domain signal, as this gives one set of components that are not time dependent $[23,24]$. Hence, the STFT method is commonly used in feature extraction of a vibration signal [25-27]. A time-domain signal is converted into a time-frequency-domain signal in the STFT method. It splits up the long time-domain signal into several segments by use of the same size of window function, and FFT coefficients are calculated for the segments. The calculated values, corresponding to the segments, are stored as a matrix. One of the advantages of STFT is that the output of the STFT can be directly used for the training of classifiers. The number of windows are used as training examples, and FFT coefficients for a window are used as features. Therefore, STFT is used for feature extraction of vibration data in this study.

The mathematical function of STFT can be expressed as:

$$
\operatorname{STFT}(\tau, w)=\int_{-\infty}^{\infty} s(t) w(t-\tau) e^{-j \omega t} d t
$$

where $s(t)$ is the original vibration signal, $w(t)$ is a windowing function, $t$ is time, and $\tau$ is time index. There are several windowing functions for STFT, such as rectangular, triangular, Hamm, Kaiser, Blackman, Gaussian, and Hann functions. The Hann windowing function is usually a good choice and is frequently employed with random data because, when compared to the impacts of other windows, it has a moderate impact on the frequency resolution and amplitude accuracy of the resulting frequency spectrum [28]. Therefore, the Hann windowing function was used in this study and can be expressed as follows:

$$
h(t)=\left\{\begin{array}{c}
0.5\left[1-\cos \left(\frac{2 \pi n}{M-1}\right)\right], 0 \leq n \leq M-1 \\
0, \text { otherwise }
\end{array}\right.
$$

where $n$ is time index and $M$ is the number of samples.

STFT is performed for the vibration signal using the librosa open-source library in Python. The output is an $n_{n_{-} f f t} \times m_{s}$ matrix, which contains complex numbers, and absolute values of the output are used. Here, $n_{-} f f t$ is the number of FFT coefficients for each window $\left(n_{-} f f t=1024\right)$, and $m_{s}$ is the number of windows. $n_{n_{-} f f t}$ is calculated as follows:

$$
n_{n_{-} f f t}=\frac{n_{-} f f t}{2}+1
$$

The hop size is 512. After feature extraction, the data were divided into $80 \%$ training data for training the model of classifiers and $20 \%$ test data for evaluating the models. Each experiment was carried out 20 times, and average values were used for the analysis.

\subsection{Machine Learning}

Since humans evolved, numerous types of tools have been used to fulfill different kinds of task in the easiest way. Different machines have been invented by humans for various tasks in human life, such as industry, computing, and so on. ML is one of them.

ML algorithms are implemented to teach machines how to handle data more fruitfully. Sometimes, by looking at the data, it is difficult to interpret the information. ML can help in this case. ML algorithms learn key features and the pattern of data, and based on this, they predict a new value. ML can be divided into several groups, such as supervised learning, unsupervised learning, semi-supervised learning, reinforcement learning, multitask learning, ensemble learning, neural networks, and instance-based learning [29]. This study belongs to supervised learning. Some popular ML classifiers used in this study are briefly described below. 
The Decision Tree Algorithm is part of the supervised machine learning family of algorithms. It is applicable to both classification and regression problems. The purpose of this algorithm is to develop a model that predicts the value of a target variable, and the decision tree solves the problem by using the tree representation, where the leaf node corresponds to a class label and characteristics are represented on the internal node of the tree [29]. The Support Vector Machine (SVM) is a part of the supervised machine learning models with accompanying learning algorithms that examine the data used for classification and regression analysis. SVM can perform non-linear classification, as well as linear classification, by implicitly mapping inputs into high-dimensional feature spaces, which is known as the kernel trick. Basically, this is used to draw lines between classes. The margins are drawn so that the space between the margin and the classes is as small as possible, reducing the classification error [30]. The K-Nearest Neighbor (KNN) algorithm is a simple supervised machine learning technique that can be used to handle classification and regression problems. It is simple to set up and comprehend, but it has the problem of being noticeably slower, as the amount of data in use grows [30]. The Random Forest algorithm is an assembled approach that generates trees using a CART (classification and regression trees) methodology to a maximum size and without pruning [31]. Bagging or Bootstrap aggregating is used when the accuracy and stability of a machine learning algorithm need to be improved. It can be used for both classification and regression. Bagging also reduces variation and aids in the management of overfitting of a decision tree [30]. XGBoost is a scalable machine learning system for tree boosting that was proposed by Chen and Guestrin in 2016 [32]. XGBoost is a well-known machine learning method that consistently outperforms other machine learning algorithms. Indeed, it has evolved into the 'state-of-the-art' machine learning technique for dealing with structured data since its debut. [33]. The Linear Discriminant Analysis (LDA) algorithm is a frequently used classification technique. The LDA method works by calculating the variance values within and between classes [34].

\section{Experimental Setup}

This study proposes a vibration-based method of early-stage bolt loosening detection and identification combined with an ML classifier for a multi-bolt structure. The experimental setup is shown in Figure 2a. The application in this study is an AC Motor, and the specification of the motor is highlighted in Table 1. The motor was fastened with four M8 $\times 1.5$ steel bolts (property class 8.8 ). The position of the bolts is shown in Figure $2 \mathrm{~b}$. According to the property class of the bolt, the maximum torque load for the M8 $\times 1.5$ bolt is $27.5 \mathrm{~N} \cdot \mathrm{m}$. Therefore, a $25 \mathrm{~N} \cdot \mathrm{m}$ torque load was considered a tightened (healthy) condition, and the torque of $19 \mathrm{~N} \cdot \mathrm{m}$ was used as a loosened (unhealthy) condition. When the decrement of a torque load is less than $6 \mathrm{~N} \cdot \mathrm{m}$ (for example, 4 or $5 \mathrm{~N} \cdot \mathrm{m}$ ), ML classifiers cannot detect bolt loosening; therefore, a $6 \mathrm{~N} \cdot \mathrm{m}$ torque load decrement was considered the beginning of the early stages of bolt loosening in this investigation.

The motor was fastened by four bolts with $25 \mathrm{~N} \cdot \mathrm{m}$ torque values that were applied with a torque wrench (Manufacturer: Tohnichi, Model: 450QL3 accuracy: $\pm 3 \%$, Figure 2c), and the motor was operated at a speed of $800 \mathrm{rpm}$ using a motor driver. For generating vibration in the structure, an unbalanced mass $(0.25 \mathrm{~kg})$ was fixed to the rotor of the motor. Following this, two accelerometer sensors (Manufacturer: Brüel \& Kjær, DK-2830 Virum, Denmark. Type: 4507B004) acquired the vibration signal of the structure for around $30 \mathrm{~s}$ and saved the signal on a laptop using an NI cDAQ-9174. The sampling rate of the acquisition system was fixed at 10,000 per second in the experiment, and LabVIEW software was used for recording the data.

Two one-axis accelerometers were used to determine the difference in prediction and detection of the early stages of bolt loosening of the multi-bolt connection system. For the first accelerometer, the axes of the sensor were mounted parallel to the axes of the rotor on the top of the motor, while the second sensor was attached to the back side of the motor, and the axes of the sensor were perpendicular to the axes of the rotor, as shown in Figure $2 \mathrm{~b}$. 
According to supervised learning, the various cases listed in Table 2 were established, and the experiment was performed for all cases, in order to collect enough vibration signals for early stage bolt-loosening detection. In addition, to improve the reliability of the proposed method, vibration signals were also acquired for motor speeds of $1000 \mathrm{rpm}$ and $1200 \mathrm{rpm}$.
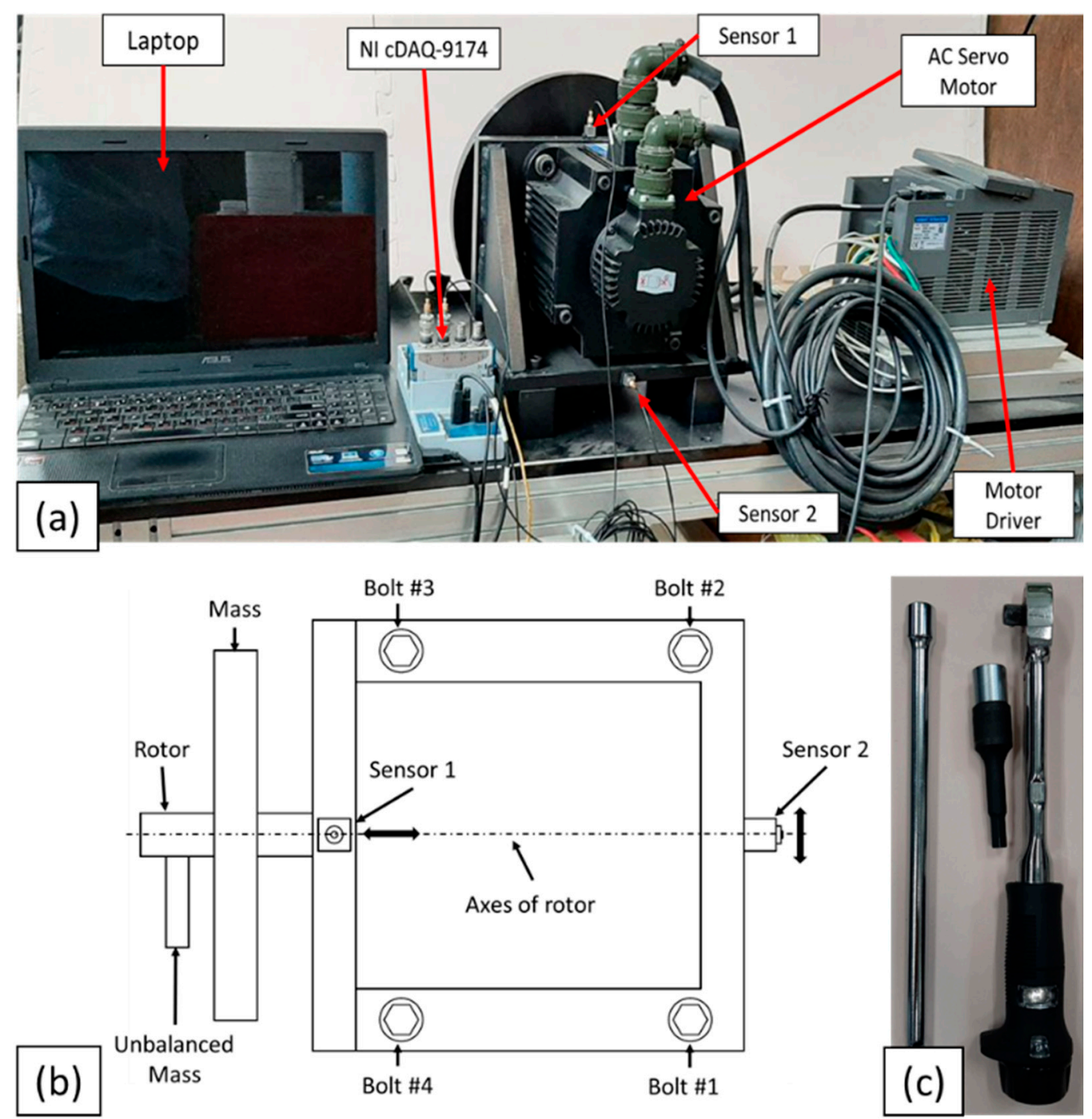

Figure 2. (a) Experimental setup; (b) scheme of top view of motor and position of bolt specimens and sensors; (c) torque wrench.

Table 1. The specification of AC Servo Motor.

\begin{tabular}{cc}
\hline Iteam & Specifications \\
\hline Name & HIGEN AC Servo Motor \\
Type & FMATN20-AB00 \\
Capacity & $1.8 \mathrm{~kW}$ \\
Torque & $11.5 \mathrm{~N} \cdot \mathrm{m}$ \\
Max. Speed & $1500 \mathrm{rpm}$ \\
Serial No. & 02003001 (ID:74) \\
\hline
\end{tabular}


Table 2. Established necessary cases for bolt-loosening detection and identification.

\begin{tabular}{|c|c|c|c|c|}
\hline Case No. & Bolt 1 & Bolt 2 & Bolt 3 & Bolt 4 \\
\hline \#1 & $\bullet$ & • & • & • \\
\hline \#2 & 0 & • & • & • \\
\hline \#3 & - & 0 & • & $\bullet$ \\
\hline$\# 4$ & • & • & 0 & 0 \\
\hline \#5 & • & • & • & 0 \\
\hline \#6 & 0 & 0 & $\bullet$ & $\bullet$ \\
\hline$\# 7$ & $\bullet$ & 0 & 0 & • \\
\hline \#8 & • & • & 0 & 0 \\
\hline$\# 9$ & 0 & $\bullet$ & 0 & • \\
\hline \#10 & 0 & $\bullet$ & $\bullet$ & 0 \\
\hline \#11 & • & 0 & • & 0 \\
\hline \#12 & 0 & 0 & 0 & 0 \\
\hline \#13 & $\bullet$ & 0 & 0 & 0 \\
\hline \#14 & 0 & • & 0 & 0 \\
\hline \#15 & 0 & 0 & • & 0 \\
\hline \#16 & 0 & 0 & 0 & $\bullet$ \\
\hline
\end{tabular}

- is tightened and $\bigcirc$ is loosened.

\section{Results and Discussion}

As mentioned above, the main goal of this study was to detect the early stages of bolt loosening of the motor and to find the best ML classification algorithm for future work on online monitoring of the motor. Therefore, the various classification algorithms listed in Table 3 were tested in the Python programming language with use of the sklearn package. Figure 3 shows the similarity of the row vibration signal between all cases. The figure shows a row of vibration signals measured by acceleration sensor 2 when the motor operating condition was $800 \mathrm{rpm}$. All signals were plotted during the timeframe of $30 \mathrm{~s}$. From the figure, it is too complicated to identify or classify which bolt is loosened or not for a human or even an experienced worker. In order to identify key features of the vibration data, STFT was performed, and spectrogram of STFT results are shown in Figure 4. The $Y$-axis of the spectrogram describes 513 FFT coefficients (features), which came from function (3), and the number of segments (windows) lays on the $X$-axis. However, classification of the data, such as finding which spectrogram belongs to which case is still complex, even for experts. However, ML classification algorithms can help to overcome this issue.

Table 3. List of classifiers for bolt-loosening detection.

\begin{tabular}{cc}
\hline Number & Classifiers \\
\hline 1 & Random Forest \\
2 & Bagged Trees \\
3 & Decision Tree \\
4 & KNeighbor \\
5 & Linear Discriminant Analysis \\
6 & Support Vector Machine \\
7 & XGBoost \\
\hline
\end{tabular}

As already introduced, in order to verify the effect of the accelerometer sensors' position on the accuracy of the ML classifiers for identification and classification of the early stage of bolt loosening in a multi-bolt connection structure, two sensors were used. Figure 5 shows the accuracy of the ML classifiers, which were used for all features (513 features) for both sensors. All classifiers had a greater accuracy for sensor 2 than sensor 1, except for the SVM classifier. According to the graph, the position of the sensor significantly affected to the accuracy of the classifiers, and the position of sensor 2 was considered the better place for identification and classification of loosening of the multi-bolt connected structure. Therefore, the rest of the results that will be discussed below are for the acceleration signal 
acquired by sensor 2 . It should be noted here that the random forest (RF), bugged trees (BT), and XGBoost classifiers showed an accuracy greater than 90 percent, while the rest of the classifiers performed with lower accuracy.
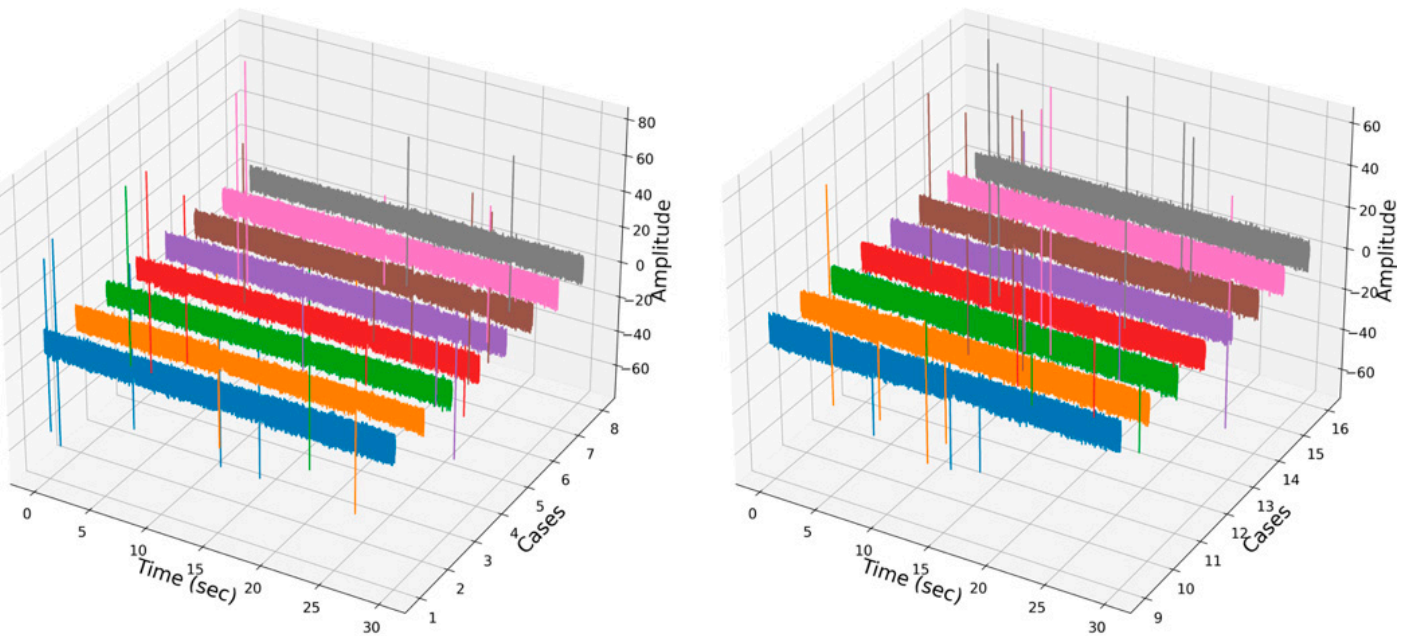

Figure 3. Rows of vibration signals of the dataset corresponding to all cases.
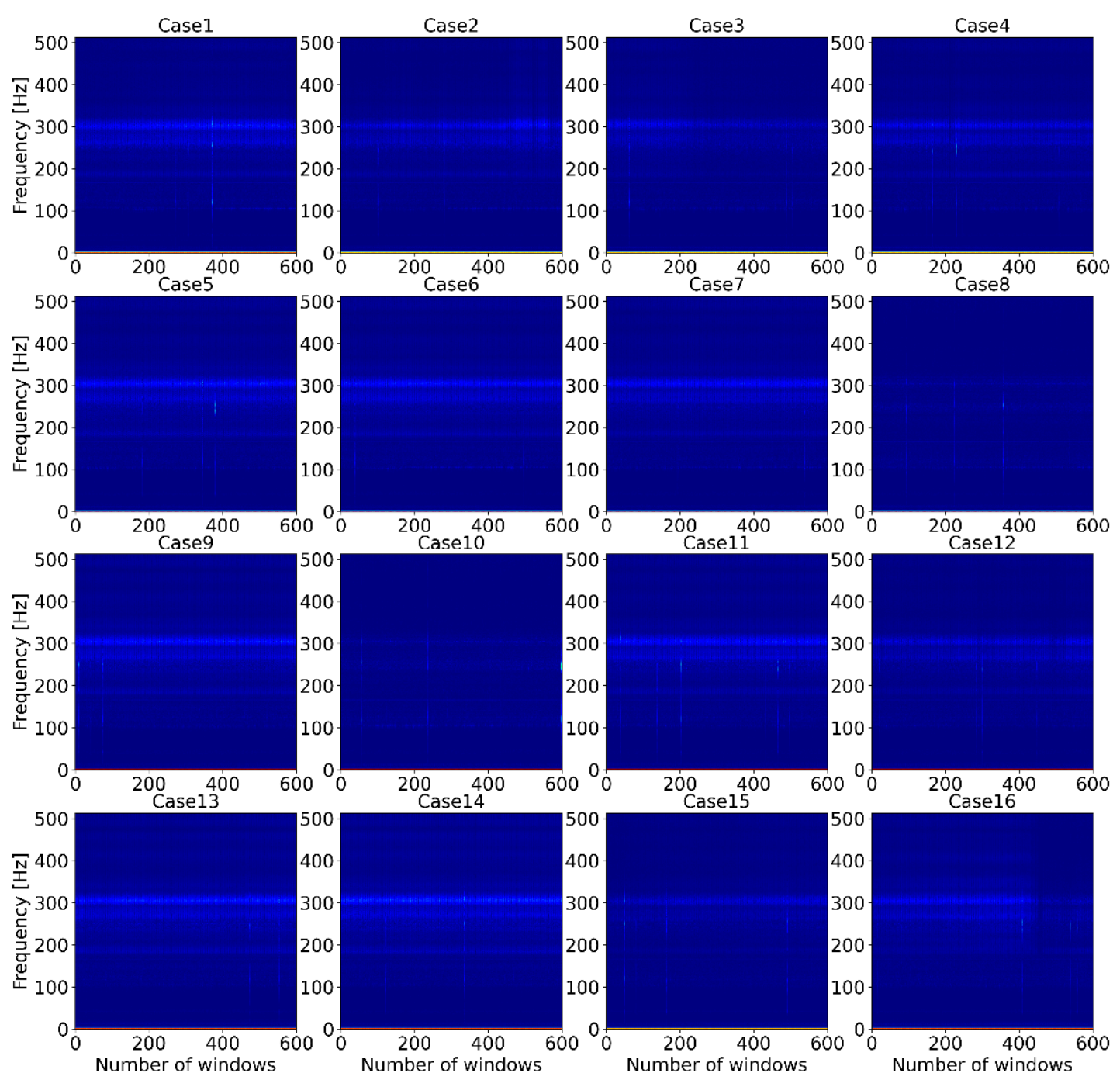

Figure 4. The spectrograms of short-time Fourier transform coefficients for all cases. 


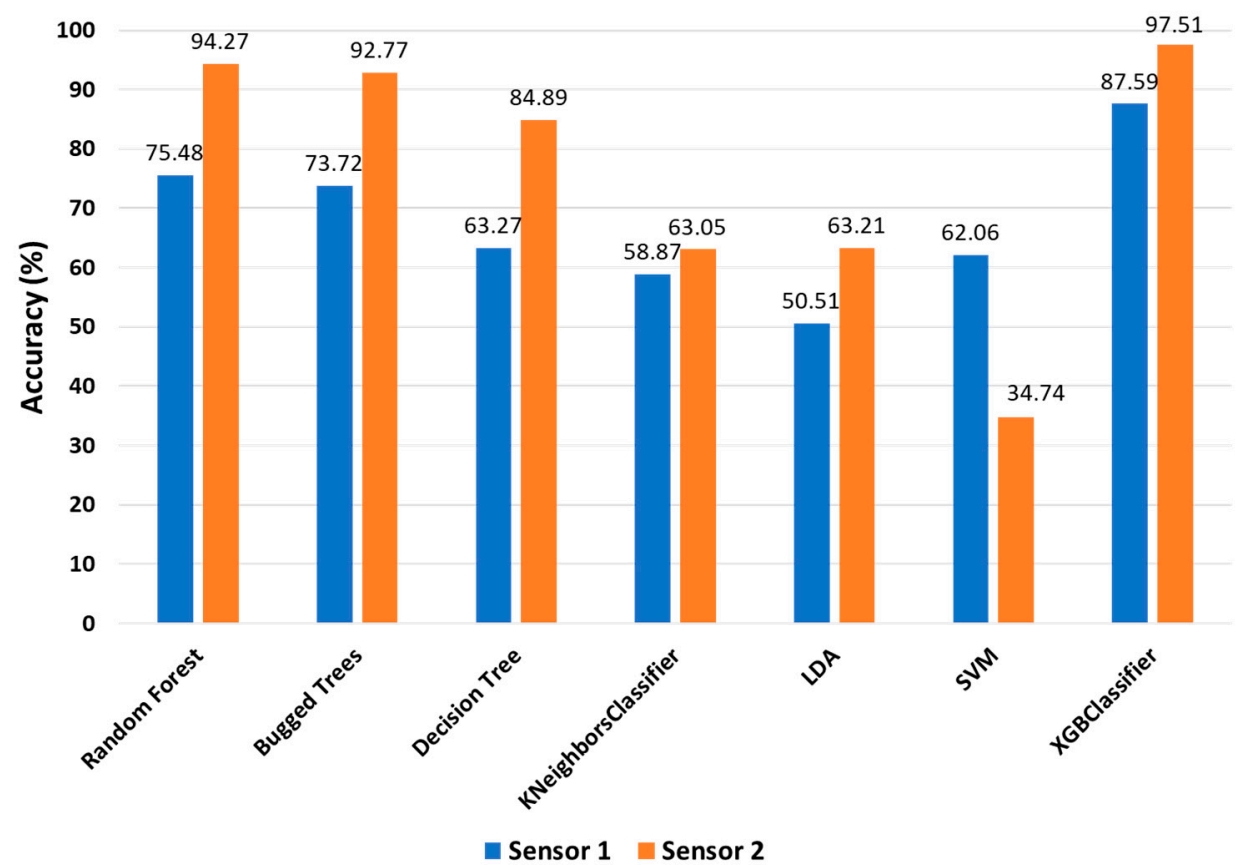

Figure 5. Accuracy of classifiers for both sensors with 513 features.

The training times of the classifiers are shown in Figure 6. According to the figure, KNeighbors (KNN) and linear discriminant analysis (LDA) classifiers required the least time for training, which is suitable for online monitoring conditions, but their accuracy was not satisfactory. The RF, BT, and XGBoost classifiers spent too much time for training, despite having the best accuracy. Hence, it was necessary to reduce the training time of the classifiers, while maintaining the accuracy.

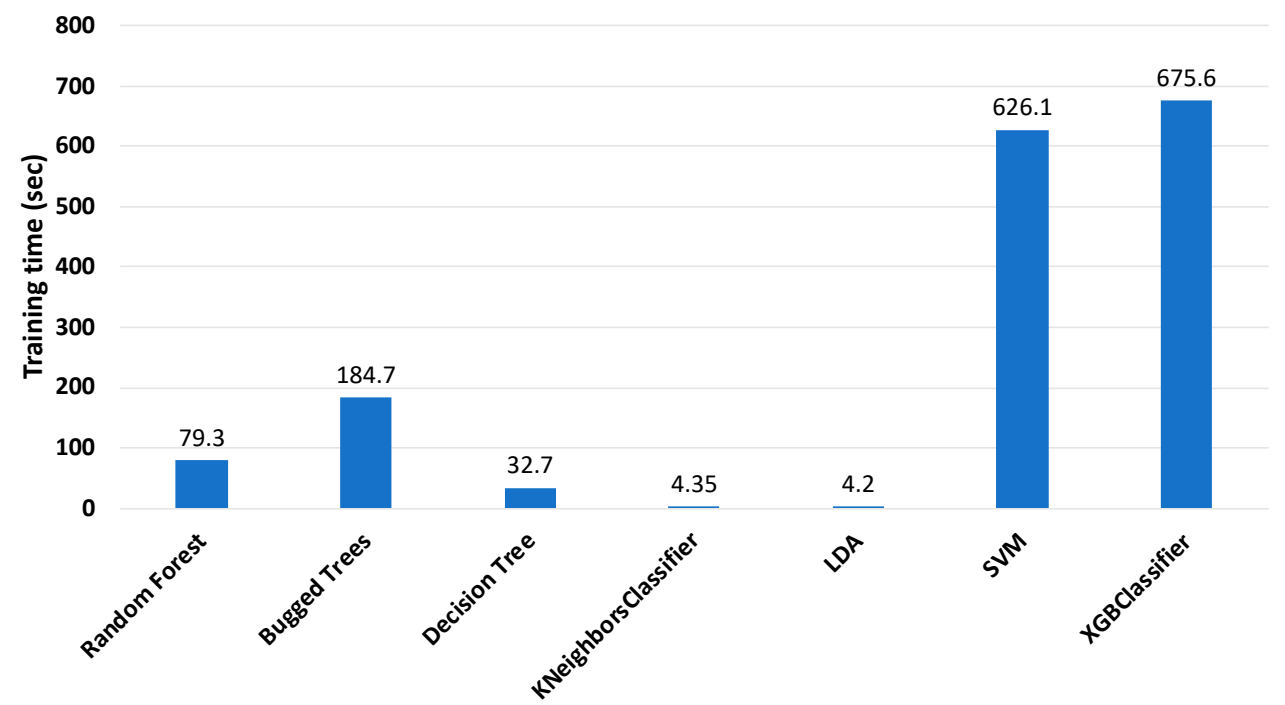

Figure 6. Training time of classifiers with 513 features (sensor 2).

To tackle this issue, feature reduction for the dataset was conducted. If the initial spectrogram of STFT results of the vibration signal was zoomed out, the key features of all the data were located between 0 and $5 \mathrm{~Hz}$ frequency, as shown in Figure 7. Therefore, the frequencies between 0 and $5 \mathrm{~Hz}$ were selected as features. Following that, the classifiers were implemented, but the accuracy of the classifiers decreased significantly. Therefore, the frequency was increased by $5 \mathrm{~Hz}$, and the classifiers were tested. This condition was repeated until satisfactory results were obtained. Consequently, when the frequency was between 0 and $25 \mathrm{~Hz}$, the classifiers had the best accuracy. Following that, in order to 
represent the differences between all cases more clearly, the spectrograms were plotted for the very beginning window (segment) of all cases.
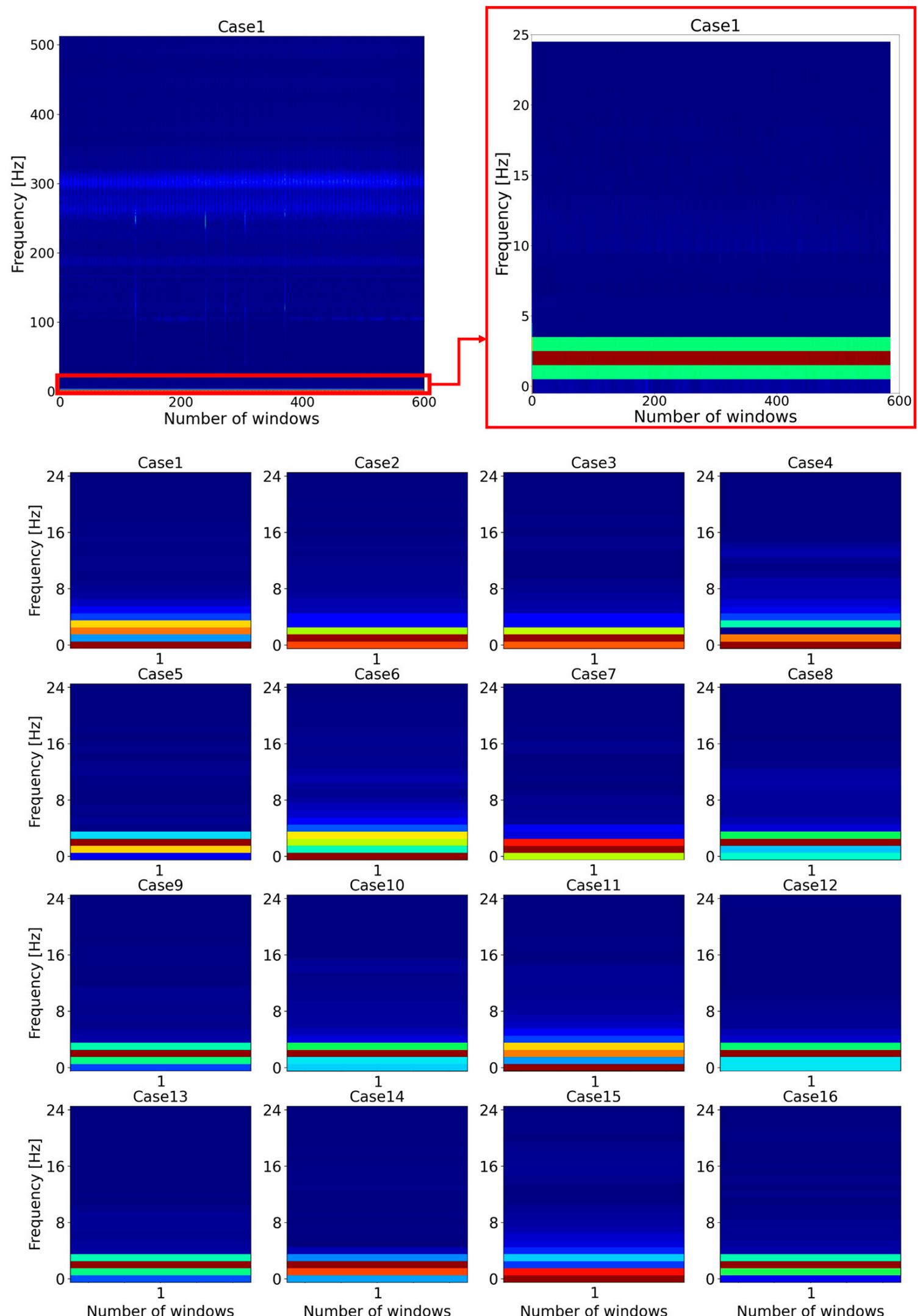

Figure 7. Representation of the feature reduction method for all cases. 
Once the feature extraction was performed, all ML classifiers were tested, and the accuracy of the classifiers with 25 features is highlighted in Figure 8. It should be mentioned here that there was a slight increase of around 1.5\% in the accuracy of the RF classifier $(95.8 \%)$, while a slight decrease of approximately $1.5 \%$ was observed in the XGBoost classifier accuracy (96.1\%). Significant growth was observed in the accuracy of DT and KNN, while there was a dramatic downward trend for LDA and SVM. However, after feature extraction, the BT classifier maintained a stable accuracy (about 92.2\%).

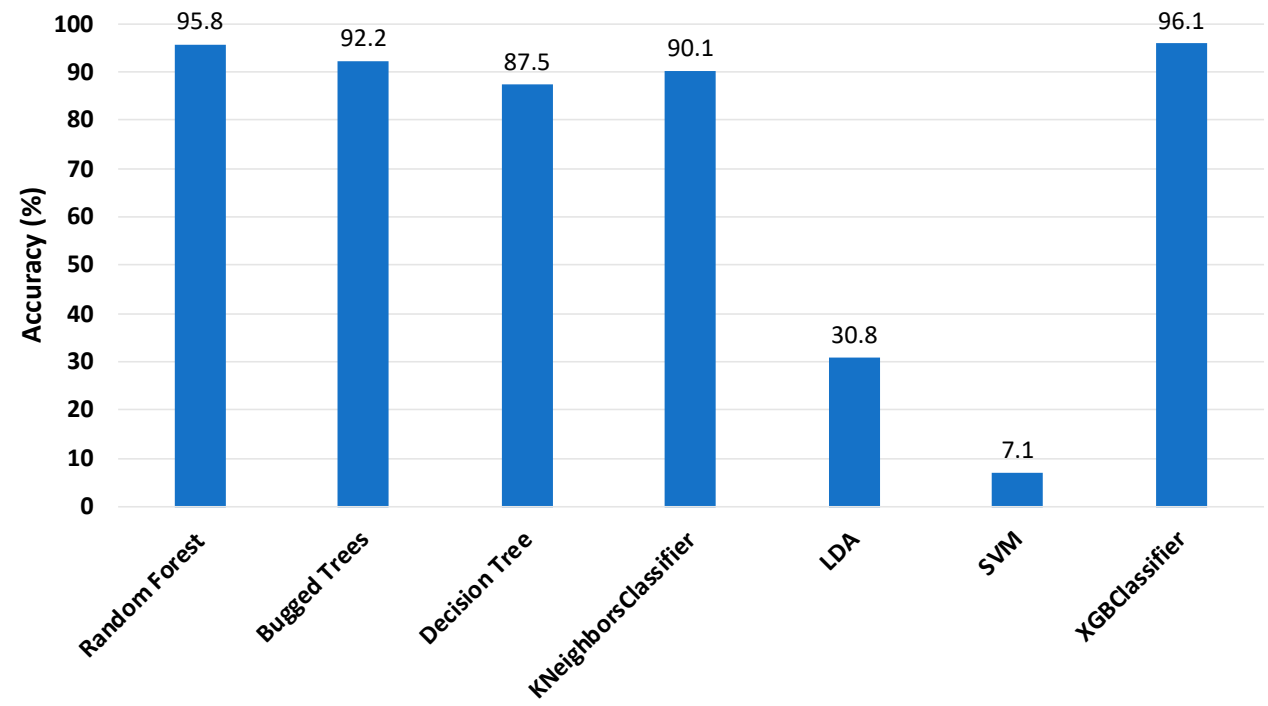

Figure 8. Accuracy of classifiers with 25 features (sensor 2).

Figure 9 shows the training time of the ML classifiers when the features of the data were reduced from 513 features to 25 features. From the graph, it is clear that there was a large reduction in the training times of the classifiers after feature reduction. As mentioned before, RF and XGboost classifiers had the highest accuracy, of approximately $96 \%$, and their training times were around 16 and $54 \mathrm{~s}$, respectively. According to the results, the RF classifier was selected, with an accuracy around $95.8 \%$ and training time of about $16 \mathrm{~s}$, for a future study on the online monitoring of the early stage of loosening of a multi-bolt structure.

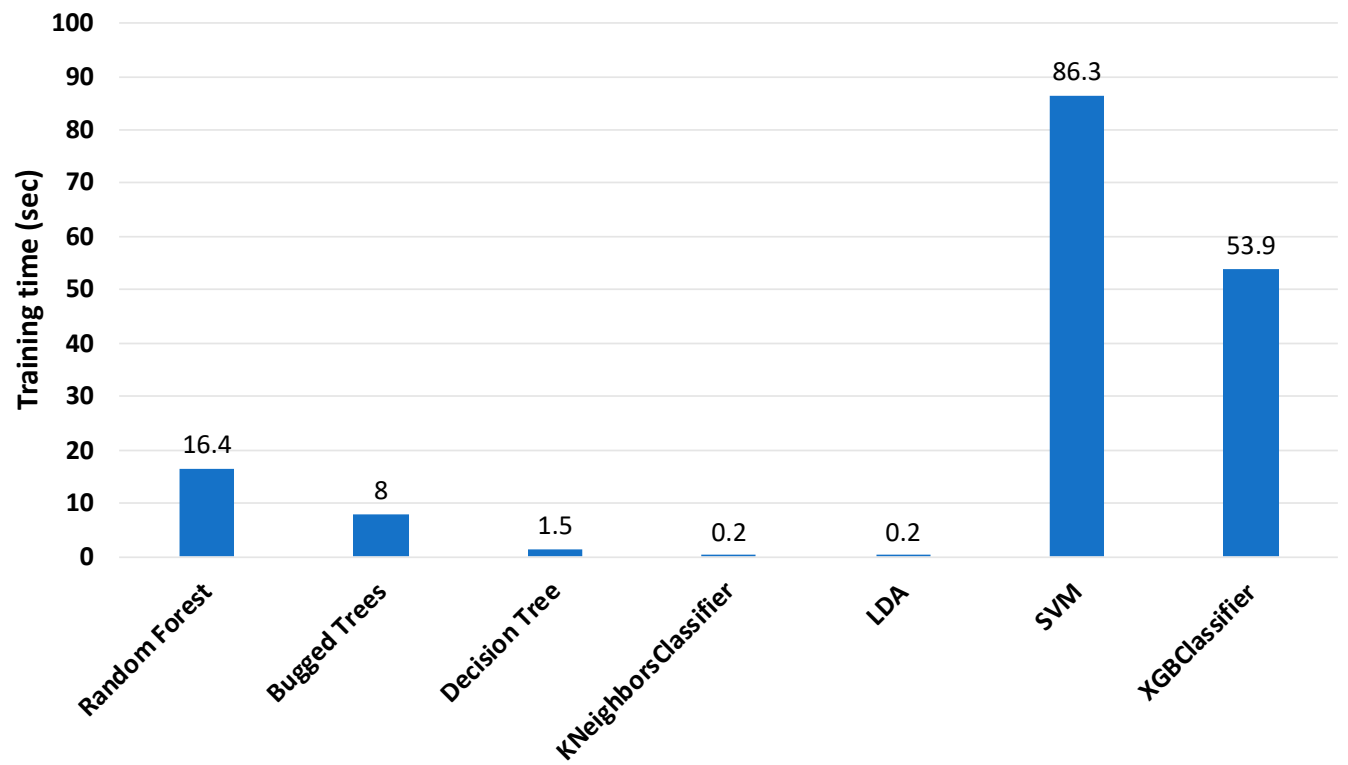

Figure 9. Training time of classifiers with 25 features (sensor 2). 
The accuracy of a classification is a synthetic variable that can only be used to assess the algorithm's overall performance, without highlighting any serious concerns about data classification. A confusion matrix can be used to analyze these concerns. A confusion matrix is also known as an error matrix, and it is a representation of statistical classification accuracy. True labels are presented in each row of the confusion matrix, while predicted labels are presented in each column [35].

Figure 10 shows the confusion matrix of the RF classifier that was selected as the classifier for a future study. The graph shows detailed information about which labels were predicted correctly and which labels failed in the prediction. In general, case 15 was the hardest label to detect, with $87.1 \%$ success, while the simplest case to classify was case 1 , with about $98.3 \%$ success. Case 5 , case 10 , and case 16 were also relatively hard to classify for the classifier, with success just above $90 \%$, compared to the rest of the cases.

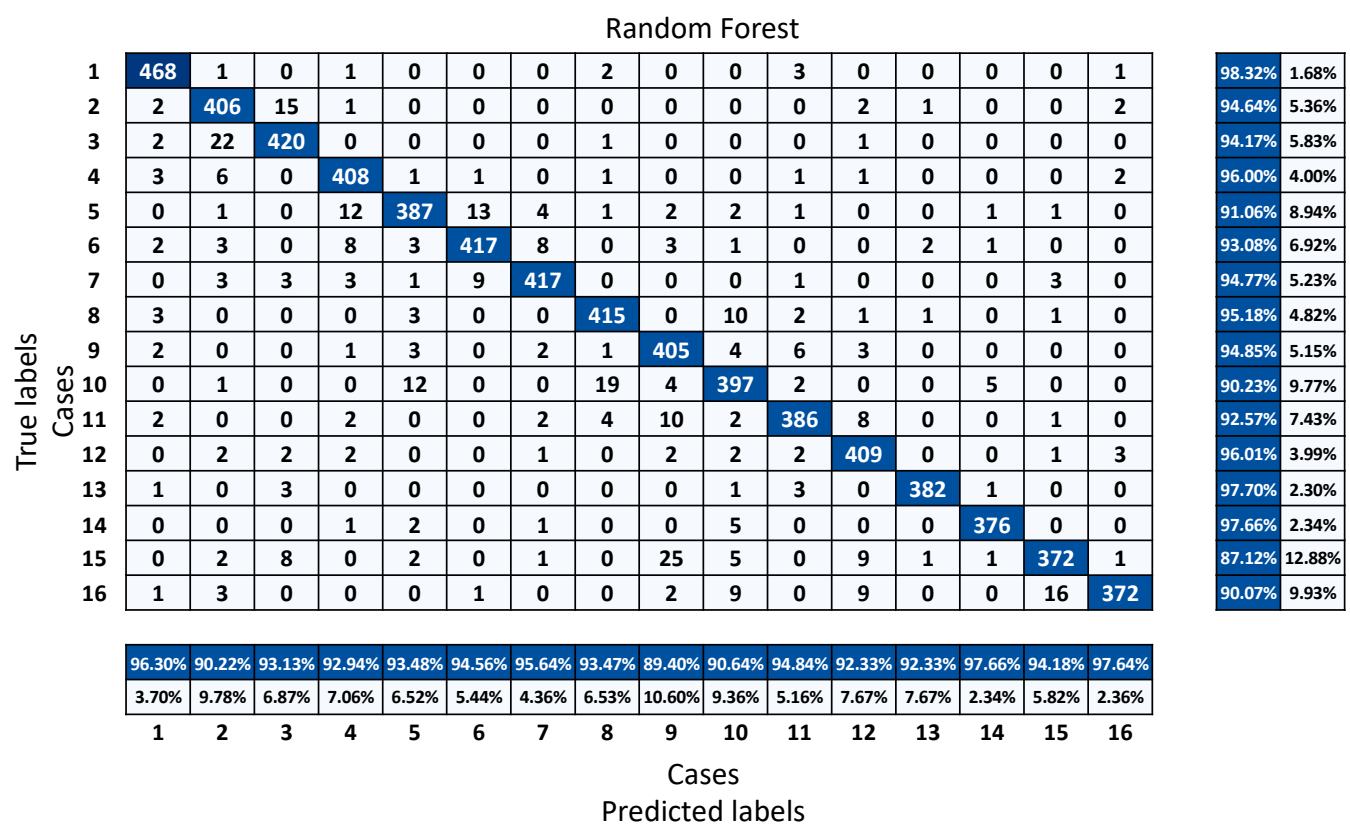

Figure 10. Confusion matrix of RF classifier.

\section{Conclusions}

In this paper, early stage bolt-loosening detection using ML classifiers for a multi-bolt structure was proposed using a vibration-based method. Then, with the help of STFT, feature extraction was performed on a row of a vibration dataset. Following this, several ML classifiers were trained and evaluated with use of the extracted dataset, to identify the best classifiers for bolt loosening detection on multi-bolt structures.

To reduce computation costs for online monitoring conditions, feature reduction was carried out. The frequencies between 0 and $25 \mathrm{~Hz}$ were selected as a feature to obtain a satisfactory accuracy for the ML classifiers. The experimental results showed that the sensor position can affect the accuracy of classifiers for early stage bolt-loosening detection of a multi-bolt structure. From the test results, the RF classifier was selected, with an accuracy around $95.8 \%$ and training time of about $16 \mathrm{~s}$, for a future study of online monitoring of loosening of a multi-bolt structure. In addition, the results of the experiments showed that it is not only possible to detect bolt loosening, but also to identify which bolt or bolts start to lose their preload.

This investigation will be broadened by conducting optimization of the sensor position to find the best place for early stage bolt loosening detection and to further develop a RF model to increase the accuracy. The investigation will be applied to online monitoring of a motor in a real condition. 
Author Contributions: Conceptualization, O.E. and K.-H.L.; methodology, O.E.; software, O.E.; validation, O.E. and K.-H.L.; formal analysis, O.E.; investigation, O.E.; resources, K.-H.L.; data curation, O.E.; writing-original draft preparation, O.E.; writing-review and editing, C.-H.L.; visualization, O.E.; supervision, C.-H.L.; project administration, C.-H.L.; funding acquisition, C.-H.L. All authors have read and agreed to the published version of the manuscript.

Funding: This research received no external funding.

Institutional Review Board Statement: Not applicable.

Informed Consent Statement: Not applicable.

Data Availability Statement: Not applicable.

Acknowledgments: This work was supported by the Korea Institute for Advancement of Technology (KIAT) grant and funded by the Korean Government (MOTIE) (P0012769, Human Resource Development Program for Industrial Innovation).

Conflicts of Interest: The authors declare no conflict of interest.

\section{References}

1. Chen, Y.; Gao, Q.; Guan, Z. Self-Loosening Failure Analysis of Bolt Joints under Vibration considering the Tightening Process. Shock Vib. 2017, 2017, 2038421. [CrossRef]

2. Park, J.H.; Kim, T.H.; Kim, J.T. Image-based bolt-loosening detection technique of bolt joint in steel bridges. Int. Conf. Adv. Exp. Struct. Eng. 2015, 1, 1-2.

3. Kong, Q.; Zhu, J.; Ho, S.C.; Song, G. Tapping and listening: A new approach to bolt looseness monitoring. Smart Mater. Struct. 2018, 27, 07LT02. [CrossRef]

4. Wang, T.; Song, G.; Liu, S.; Li, Y.; Xiao, H. Review of bolted connection monitoring. Int. J. Distrib. Sens. Netw. $2013,9,871213$. [CrossRef]

5. Pham, H.C.; Huyunh, T.C. Bolt-Loosening Monitoring Framework Using an Image-Based Deep Learning and Graphical Model. Sensors 2020, 20, 3382. [CrossRef]

6. $\quad$ Park, J.H.; Huynh, T.C.; Choi, S.H.; Kim, J.T. Vision-based technique for bolt-loosening detection in wind turbine tower. Wind Struct 2015, 21, 709-726. [CrossRef]

7. Cha, Y.J.; You, K.; Choi, W. Vision-based detection of loosened bolts using the Hough transform and support vector machines. Autom. Constr. 2016, 71, 181-188. [CrossRef]

8. Lee, S.Y.; Huynh, T.C.; Park, J.H.; Kim, J.T. Bolt-Loosening Detection using Vision-Based Deep Learning Algorithm and Image Processing Method. J. Comput. Struct. Eng. Inst. Korea 2019, 32, 265-272. [CrossRef]

9. Zhang, Y.; Sun, X.; Loh, K.J.; Su, W.; Xue, Z.; Zhao, X. Autonomous bolt loosening detection using deep learning. Struct. Health Monit. 2020, 19, 105-122. [CrossRef]

10. Zhao, X.; Zhang, Y.; Wang, N. Bolt loosening angle detection technology using deep learning. Struct. Control Health Monit. 2019, 26, e2292. [CrossRef]

11. Nichols, J.M.; Nichols, C.J.; Todd, M.D.; Seaver, M.; Trickey, S.T.; Virgin, L.N. Use of data-driven phase space models in assessing the strength of a bolted connection in a composite beam. Smart Mater. Struct. 2004, 13, 241-250. [CrossRef]

12. Razi, P.; Esmaeel, R.A.; Taheri, F. Improvement of a vibration-based damage detection approach for health monitoring of bolted flange joints in pipelines. Struct. Health Monit. 2013, 12, 207-224. [CrossRef]

13. Chaki, S.; Corneloup, G.; Lillamand, I.; Walaszek, H. Combination of longitudinal and transverse ultrasonic waves for in situ control of the tightening of bolts. J. Press. Vessel Technol. Trans. ASME 2007, 129, 383-390. [CrossRef]

14. Jhang, K.Y.; Quan, H.H.; Ha, J.; Kim, N.Y. Estimation of clamping force in high-tension bolts through ultrasonic velocity measurement. Ultrasonics 2006, 44, 1339-1342. [CrossRef]

15. Bhalla, S.; Vittal, P.A.; Veljkovic, M. Piezo-impedance transducers for residual fatigue life assessment of bolted steel joints. Struct. Health Monit. 2012, 11, 733-750. [CrossRef]

16. Ritdumrongkul, S.; Abe, M.; Fujino, Y.; Miyashita, T. Quantitative health monitoring of bolted joints using a piezoceramic actuator-sensor. Smart Mater. Struct. 2004, 13, 20-29. [CrossRef]

17. Wang, F.; Song, G. Bolt early looseness monitoring using modified vibro-acoustic modulation by time-reversal. Mech. Syst. Signal Process. 2019, 130, 349-360. [CrossRef]

18. Wang, F.; Ho, S.C.M.; Huo, L.; Song, G. A Novel Fractal Contact-Electromechanical Impedance Model for Quantitative Monitoring of Bolted Joint Looseness. IEEE Access 2018, 6, 40212-40220. [CrossRef]

19. He, K.; Zhu, W.D. Detecting loosening of bolted connections in a pipeline using changes in natural frequencies. J. Vib. Acoust. Trans. ASME 2014, 136, 1-8. [CrossRef]

20. Sejdić, E.; Djurović, I.; Jiang, J. Time-frequency feature representation using energy concentration: An overview of recent advances. Digit. Signal Process. 2009, 19, 153-183. [CrossRef] 
21. Chen, Z.; Li, C.; Sanchez, R. Gearbox Fault Identification and Classification with Convolutional Neural Networks. Shock Vib. 2015, 2015, 390134. [CrossRef]

22. Ma, S.; Chu, F. Ensemble deep learning-based fault diagnosis of rotor bearing systems. Comput. Ind. 2019, 105, 143-152. [CrossRef]

23. Liang, L.; Wen, H.; Liu, F.; Li, G.; Li, M. Feature extraction of impulse faults for vibration signals based on sparse non-negative tensor factorization. Appl. Sci. 2019, 9, 3642. [CrossRef]

24. Dass, S.; Holi, M.S.; Rajan, K.S.; Prof, A. A Comparative Study on FFT, STFT and WT for the Analysis of Auditory Evoked Potentials. IJERT 2013, 2, 636-641.

25. Indra Devi, M.; Rajaram, R.; Selvakuberan, K. Machine learning techniques for automated web page classification using URL features. In Proceedings of the International Conference on Computational Intelligence and Multimedia Applications (ICCIMA 2007), Sivakasi, India, 13-15 December 2007; Volume 2, pp. 116-118.

26. Shovon, T.H.; Al Nazi, Z.; Dash, S.; Hossain, M.F. Classification of motor imagery EEG signals with multi-input convolutional neural network by augmenting STFT. In Proceedings of the 2019 5th International Conference on Advances in Electrical Engineering (ICAEE), Dhaka, Bangladesh, 26-28 September 2019; pp. 398-403.

27. Huang, J.; Chen, B.; Yao, B.; He, W. ECG Arrhythmia Classification Using STFT-Based Spectrogram and Convolutional Neural Network. IEEE Access 2019, 7, 92871-92880. [CrossRef]

28. Encyclopedia of Vibration I ScienceDirect. Available online: https:/ /www.sciencedirect.com/referencework/9780122270857/ encyclopedia-of-vibration (accessed on 20 January 2022).

29. Rao, B. Machine Learning Algorithms: A Review. Int. J. Comput. Sci. Inf. Technol. 2016, 7, 1174-1179.

30. Mahesh, B. Machine Learning Algorithms-A Review. Int. J. Sci. Res. 2020, 9, 381-386.

31. Yang, B.S.; Di, X.; Han, T. Random forests classifier for machine fault diagnosis. J. Mech. Sci. Technol. 2008, $22,1716-1725$. [CrossRef]

32. Tianqi Chen, C.G. XGBoost: A Scalable Tree Boosting System. J. Assoc. Phys. India 2016, 42, 665.

33. Zhang, D.; Qian, L.; Mao, B.; Huang, C.; Huang, B.; Si, Y. A Data-Driven Design for Fault Detection of Wind Turbines Using Random Forests and XGboost. IEEE Access 2018, 6, 21020-21031. [CrossRef]

34. Zhao, W.; Chellappa, R.; Nandhakumar, N. Empirical performance analysis of linear discriminant classifier. In Proceedings of the 1998 IEEE Computer Society Conference on Computer Vision and Pattern Recognition, Santa Barbara, CA, USA, 25 June 1998; pp. 164-169.

35. Casoli, P.; Pastori, M.; Scolari, F.; Rundo, M. A vibration signal-based method for fault identification and classification in hydraulic axial piston pumps. Energies 2019, 12, 953. [CrossRef] 